\title{
ON A DIRECTIONALLY REINFORCED RANDOM WALK
}

\author{
ARKA P. GHOSH, REZA RASTEGAR, AND ALEXANDER ROITERSHTEIN
}

(Communicated by Mark M. Meerschaert)

\begin{abstract}
We consider a generalized version of a directionally reinforced random walk, which was originally introduced by Mauldin, Monticino, and von Weizsäcker. Our main result is a stable limit theorem for the position of the random walk in higher dimensions. This extends a result of Horváth and Shao that was previously obtained in dimension one only (however, in a more stringent functional form).
\end{abstract}

\section{INTRODUCTION}

In this paper we study the following directionally reinforced random walk. Fix $d \in \mathbb{N}$ and a finite set $U$ of distinct unit vectors in $\mathbb{R}^{d}$ (see Remark 2.8 at the end of Section 2 below, where a suitable alternative Markovian setup in a general state space is discussed). The vectors in $U$ serve as feasible directions for the motion of the random walk. To avoid trivialities we assume that $U$ contains at least two elements. Let $X_{t} \in \mathbb{R}^{d}$ denote the position of the random walk at time $t$. Throughout the paper we assume that $X_{0}=0$. The random walk changes its direction at random times

$$
s_{1}:=0<s_{2}<s_{3}<s_{4}<\ldots
$$

We assume that the time intervals

$$
T_{n}:=s_{n+1}-s_{n}, \quad n \in \mathbb{N},
$$

are independent and identically distributed. Let $\eta_{n} \in U$ be the direction of the walk during time interval $\left[s_{n}, s_{n+1}\right)$. We assume that $\eta:=\left(\eta_{n}\right)_{n \geq 1}$ is an irreducible stationary Markov chain on $U$ which is, furthermore, independent of $\left(s_{n}\right)_{n \in \mathbb{N}}$.

For $t>0$, let $N_{t}:=\sup \left\{k \geq 1: s_{k} \leq t\right\}$ be the number of times that the walker changes direction before time $t>0$. Then

$$
X_{t}=\sum_{i=1}^{N_{t}-1} \eta_{i} T_{i}+\left(t-s_{N_{t}}\right) \eta_{N_{t}} .
$$

Notice that $N_{t} \geq 1$ with probability one, due to the convention $s_{1}=0$ that we have made.

The continuous time random walk $\left(X_{t}\right)_{t \geq 0}$ defined above is essentially the model introduced by Mauldin, Monticino, and von Weizsäcker in [20] and further studied by Horváth and Shao in 13 and by Siegmund-Schultzea and von Weizsäcker in 25]. The technical difference between our model and the variant which has been studied in [13] is that in the latter the next direction of the motion is chosen uniformly

Received by the editors May 31, 2012 and, in revised form, September 19, 2012.

2010 Mathematics Subject Classification. Primary 60F05, 60F15; Secondary 60J25, 70 B05. 
from the available set of "fresh directions", while we do not impose any restrictions on the transition kernel of $\eta$ besides irreducibility.

The original model proposed in 20] was inspired by certain phenomena that occur in ocean surface waves (cf. [30]) and was designed to reproduce the same features within a probabilistic framework. The main topic of [20] and [25] is recurrencetransience criteria. Horváth and Shao in 13 studied scaling limits of the random walk in different regimes, answering some of the questions which have been posed in 20 .

We remark that somewhat related random walk models have been considered by Allaart and Monticino in [1,2 and by Gruber and Schweizer in [12. In the context of random walks in random environment, a similar in spirit model of persistent random walks was introduced by Szász and Tóth in [28, 29]. The common feature of "generic versions" of all models mentioned above is that the underlying random motion has a tendency to persist in its current direction.

Closely related to persistent random walks are recurrent "random flights" models where changes of the direction of the random motion follow a Poisson random clock. These models can be traced back to Pearson's random walk [10, 15] and the Goldstein-Kac one-dimensional "telegraph process" [16, 23] . Random flights have been intensively studied since the introduction of the telegraph process in the early 1950s; see for instance $[11,17-19,22,26]$ and the references therein for a representative sample. An introductory part of 18 provides a short authoritative and up-to-date survey of the field. We remark that, somewhat contrary to directionally reinforced random walks, the main focus of the research in this area is in finding an explicit form of limiting distributions for these processes.

In statistical physics, the model (1.1) with i.i.d. jump vectors $\eta_{i}$ is a certain kind of coupled continuous time random walk. Limit theorems for coupled continuous time random walks have been developed in [5, 14, 21, 27, focusing on the case where the waiting times have infinite mean.

The main goal of this paper is to prove stable limit theorems for the directionally reinforced random walk in arbitrary dimension $d \geq 1$. In addition, we extend some limit results of 13 to our setting and also complement them by suitable laws of iterated logarithm. Our proofs can be easily carried over to a setup where the set of feasible directions $U$ is not finite, but is rather supported (under the stationary law of the process) on a general Borel subset of the unit sphere; see Remark 2.8 below for more details.

Our results are stated in Section 2 whereas the proofs are contained in Section 3 . The non-Gaussian limit theorems for the position of the random walk in higher dimensions, stated in Theorems 2.5] and 2.6. constitute the main contribution of this paper.

\section{Statement of MAin RESUlts}

We first introduce some notation. For a vector $x=\left(x_{1}, \ldots, x_{d}\right) \in \mathbb{R}^{d}$ let $\|x\|=\max _{i}\left|x_{i}\right|$. For (possibly, random) functions $f, g: \mathbb{R}_{+}$(or $\mathbb{N}$ ) $\rightarrow \mathbb{R}$, write $f \sim g$ and $f(t)=o(g(t))$ to indicate that, respectively, $\lim _{t \rightarrow \infty} f(t) / g(t)=1$ and $\lim _{t \rightarrow \infty} f(t) / g(t)=0$, a.s. Let $\pi=\left(\pi_{v}\right)_{v \in U} \in \mathbb{R}^{|U|}$ be the unique stationary distribution of the Markov chain $\eta$ and let

$$
\mu=\sum_{v \in U} \pi_{v} v
$$


Thus $\mu=E\left(\eta_{n}\right) \in \mathbb{R}^{d}$ for each $n \in \mathbb{N}$.

The following theorem shows that a strong law of large numbers holds for $X_{t}$ and that, under a suitable second moment condition, the sample paths of the random walk are uniformly close to the sample paths of a drifted Brownian motion. We have:

\section{Theorem 2.1.}

(a) Suppose that $E\left(T_{1}^{p}\right)<\infty$ for some constant $p \in(1,2)$. Then,

$$
\left\|X_{t}-\mu t\right\|=o\left(t^{1 / p}\right) .
$$

(b) If $E\left(T_{1}^{p}\right)<\infty$ for some constant $p>2$, then (in an enlarged, if needed, probability space) there exist a process $\widehat{X}=\left(\widehat{X}_{t}\right)_{t \geq 0}$ distributed as $X$ and a Brownian motion $\left(W_{t}\right)_{t \geq 0}$ in $\mathbb{R}^{d}$ such that

$$
\sup _{0 \leq t \leq T}\left\|\widehat{X}_{t}-\mu t-W_{t}\right\|=o\left(T^{1 / p}\right) .
$$

Remark 2.2. The results stated in Theorem 2.1 as well as in Theorem 2.3 below are essentially due to 13 . In fact, the original proofs can be adapted to our more general setup. However, the proofs we give in Section 3 are shorter and somewhat simpler than the original ones. Furthermore, our proofs can easily be seen working for the general Markov chain setup described in Remark 2.8 below.

The second part of Theorem 2.1 implies the invariance principle for $\left(X_{n t}-\mu n t\right)$ with the usual normalization $\sqrt{n}$. We next state an invariance principle and the corresponding law of iterated logarithm under a slightly more relaxed moment condition. Let $D\left(\mathbb{R}^{d}\right)$ denote the set of $\mathbb{R}^{d}$-valued càdlàg functions on $[0,1]$ equipped with the Skorokhod $J_{1}$-topology. We use the notation $\Rightarrow$ to denote the weak convergence in $D\left(\mathbb{R}^{d}\right)$. We have:

Theorem 2.3. For $n \in \mathbb{N}$, define a process $S_{n}$ in $D\left(\mathbb{R}^{d}\right)$ by setting

$$
S_{n}(t)=\frac{X_{n t}-\mu n t}{\sqrt{n}}, \quad t \in[0,1] .
$$

If $E\left(T_{1}^{2}\right)<\infty$, then

(a) $S_{n} \Rightarrow W$, where $W=\left(W_{t}\right)_{t \geq 0}$ is a (possibly degenerate, but not identically equal to zero) d-dimensional Brownian motion.

(b) For every $x \in \operatorname{Span}(U) \subset \mathbb{R}^{d}$, there is a constant $K(x) \in(0, \infty)$ such that

$$
\limsup _{t \rightarrow \infty} \frac{\left(X_{t}-\mu t\right) \cdot x}{\sqrt{t \ln \ln t}}=K(x) .
$$

Furthermore, a similar statement holds for the liminf.

We next consider the case when $E\left(T_{1}^{2}\right)=\infty$ and $T_{1}$ is in the domain of attraction of a stable law. Namely, for the rest of our results we impose the following assumption. Recall that a function $h: \mathbb{R}_{+} \rightarrow \mathbb{R}$ is said to be regularly varying of index $\alpha \in \mathbb{R}$ if $h(t)=t^{\alpha} L(t)$ for some $L: \mathbb{R}_{+} \rightarrow \mathbb{R}$ such that $L(\lambda t) \sim L(t)$ for all $\lambda>0$. We will denote the set of all regularly varying functions of index $\alpha$ by $\mathcal{R}_{\alpha}$.

Assumption 2.4. For some $\alpha \in(0,2]$ there exists a function $h \in \mathcal{R}_{\alpha}$ such that $\lim _{t \rightarrow \infty} h(t) \cdot P\left(T_{1}>t\right) \in(0, \infty)$. 
For $t>0$ let

$$
a_{t}= \begin{cases}\inf \left\{s>0: t \cdot P\left(T_{1}>s\right) \leq 1\right\} & \text { if } \quad \alpha<2, \\ \inf \left\{s>0: t s^{-2} \cdot E\left(T_{1}^{2} ; T_{1} \leq s\right) \leq 1\right\} & \text { if } \quad \alpha=2 .\end{cases}
$$

If $h(t) \in \mathcal{R}_{\alpha}$ with $\alpha \in(1,2]$ (and hence $E\left(T_{1}\right)<\infty$ ), one can obtain the following analogue of Theorem 2.3 . It turns out that also in this case the functional limit theorem and the law of iterated logarithm for $X_{t}$ inherit the structure of the corresponding statements for the partial sums of i.i.d. variables $\sum_{k=1}^{n} T_{k}$.

Theorem 2.5. Let Assumption 2.4 hold with $\alpha \in(1,2]$. Let

$$
S_{t}:=\frac{X_{t}-\mu t}{a_{t}}, \quad t>0 .
$$

We have:

(a) If $\alpha \in(1,2)$, then

(i) $S_{t}$ converges weakly to a non-degenerate multivariate stable law in $\mathbb{R}^{d}$.

(ii) For every $x \in \operatorname{Span}(U) \subset \mathbb{R}^{d}$ such that $x \cdot u>0$ for some $u \in U$,

$$
\limsup _{t \rightarrow \infty} \frac{\left(X_{t}-\mu t\right) \cdot x}{a_{t} \cdot(\ln t)^{1 / \alpha+\varepsilon}}=\left\{\begin{array}{ll}
0 & \text { if } \varepsilon>0, \\
\infty & \text { if } \varepsilon<0
\end{array} \quad\right. \text { a.s. }
$$

In particular,

$$
\limsup _{t \rightarrow \infty}\left\{\frac{\left(X_{t}-\mu t\right) \cdot x}{a_{t}}\right\}^{1 / \ln \ln t}=e^{1 / \alpha} \quad \text { a.s. }
$$

(b) If $\alpha=2$ and $E\left(T_{1}^{2}\right)=\infty$, then $S_{t}$ converges weakly to a non-degenerate multivariate Gaussian distribution in $\mathbb{R}^{d}$.

For $\alpha \in(0,1)$ we have the following limit theorem.

Theorem 2.6. Let Assumption 2.4 hold with $\alpha \in(0,1)$. Then $\frac{X_{t}}{t}$ converges weakly in $\mathbb{R}^{d}$ to a non-degenerate limit.

Remark 2.7. The limiting random law in the statement of Theorem 2.6 is specified in (3.24) below. The stable limit laws for $X_{t}$ stated in Theorems 2.5 and Theorem 2.6 are extensions of corresponding one-dimensional results in [13. The latter however are obtained in [13] in a more stringent functional form. The law of iterated logarithm given in Theorem 2.5] appears to be new even for $d=1$.

Remark 2.8. Recall the Markov chain $\eta=\left(\eta_{n}\right)_{n \geq 0}$ which records successive directions of the random walk. Let $\mathcal{S}^{d-1}$ denote the $d$-dimensional unit sphere and let $\mathcal{T}_{d}$ denote the $\sigma$-algebra of the Borel sets of $\mathcal{S}^{d-1}$. Denote by $H(x, A)$ the transition kernel of $\eta$ on $\left(\mathcal{S}^{d-1}, \mathcal{T}_{d}\right)$. We remark that

(i) All the results stated in this section remain true for an arbitrary (not stationary) initial distribution of the Markov chain $\eta$.

(ii) The proofs of our results given in Section 3 rest on the exploiting of a regenerative (renewal) structure associated with $\eta$, i.e. on the use of random times $\tau_{n}$ which are introduced below in Section 3.1. It is then not hard to verify that all the results stated in this section, with the only exception being the generalized law of iterated logarithm given in part (a)-(ii) of Theorem 2.5, remain true for a class of regenerative (in the sense of [3]) Markov chains $\eta$ whose stationary distribution are supported on general 
Borel subsets of $\mathcal{S}^{d-1}$ rather than on a finite set $U \subset \mathcal{S}^{d-1}$. For instance, the following strong version of the classical Doeblin's conditions is sufficient for our purposes:

- There exist a constant $c_{r}>1$ and a probability measure $\psi$ on $\left(\mathcal{S}^{d-1}, \mathcal{T}_{d}\right)$ such that

$$
c_{r}^{-1} \psi(A)<H(x, A)<c_{r} \psi(A) \quad \forall x \in \mathcal{S}, A \in \mathcal{T}_{d} .
$$

A regenerative (renewal) structure for Markov chains which satisfies Doeblin's condition is described in [3]. Due to the fact that under the assumption (2.4) the kernel $H(x, A)$ is dominated uniformly from above and below by a probability measure $\psi$, such Markov chains share two key features with finite-state Markov chains. Namely, 1) the exponential bound stated in (3.2) holds for the renewal times which are defined in [3]; and 2) $c_{r}^{-1}<P_{x}(A) / P_{y}(A)<c_{r}$ for any non-null event $A \in \mathcal{T}_{d}$ and almost every state $x, y \in \mathcal{S}^{d-1}$ (with respect to the stationary law). Here $P_{x}$ stands for the law of the Markov chain $\eta$ starting from the initial state $x \in \mathcal{S}^{d-1}$. Once these two crucial properties are verified, our proofs (except only the proof of part (a)-(ii) of Theorem 2.5) work nearly verbatim for directionally reinforced random walks governed by a Markov chain $\eta$ which satisfies condition (2.4).

\section{Proofs}

This section is devoted to the proof of the results stated in Section 2 above. Some preliminary observations are stated in Section 3.1 below. The proof of Theorem 2.1 is contained in Section [3.2. Theorems 2.3 and 2.5 are proved in Section 3.3 and Section 3.4 respectively. Finally, the proof of Theorem 2.6 is given in Section 3.5 .

3.1. Preliminaries. Our approach relies on the use of a renewal structure which is induced on the paths of the random walk by the cycles of the underlying Markov chain $\eta$. To define the renewal structure, set $\tau_{0}=0$ and let

$$
\tau_{i+1}=\inf \left\{j>\tau_{i}: \eta_{j}=u_{1}\right\}, \quad i \geq 0 .
$$

Thus, for $i \geq 1, \tau_{i}$ are steps when the Markov chain $\eta$ visits the distinguished state $u_{1}$. Correspondingly, $s_{\tau_{i}}$ are successive times when the random walk chooses $u_{1}$ as the direction of its motion. Recall $N_{t}$ from Section 1 (see a few lines preceding (1.1)). Denote by $c(t)$ the number of times that the walker chooses direction $u_{1}$ before time $t>0$. That is,

$$
c(t):=\sup \left\{i \geq 0: s_{\tau_{i}} \leq t\right\}=\sum_{j=1}^{N_{t}} \mathbf{1}_{\left\{\eta_{j}=u_{1}\right\}},
$$

where $\mathbf{1}_{A}$ stands for the indicator function of an event $A$. Notice that $N_{t}$ is the unique mapping from $\mathbb{R}_{+}$to $\mathbb{Z}_{+}$which has the following property:

$$
s_{N_{t}} \leq t<s_{N_{t}+1} \quad \text { and } \quad \tau_{c(t)} \leq N_{t}<\tau_{c(t)+1} .
$$

For $i \geq 0$, let $\xi_{i}=\sum_{j=\tau_{i}+1}^{\tau_{i+1}} T_{j} \eta_{j}$. Then

$$
X_{t}=\xi_{0}+\sum_{i=1}^{c(t)-1} \xi_{i}+\sum_{j=\tau_{c(t)}+1}^{N_{t}} T_{j} \eta_{j}+\left(t-s_{N_{t}}\right) \cdot \eta_{N_{t}} .
$$


The strong Markov property implies that the pairs $\left(\xi_{i}, \tau_{i+1}-\tau_{i}\right)_{i \in \mathbb{N}}$ form an i.i.d. sequence which is independent of $\left(\xi_{0}, \tau_{1}\right)$. Furthermore, since $\eta$ is an irreducible finite-state Markov chain, there exist positive constants $K_{1}, K_{2}>0$ such that the inequality

$$
P\left(\tau_{i+1}-\tau_{i}>t\right) \leq K_{1} e^{-K_{2} t}
$$

holds uniformly for all reals $t \geq 0$ and all integers $i \geq 0$.

We next list some direct consequences of the law of large numbers that will be frequently exploited in the subsequent proofs. Let $v(n)$ be the number of times that the Markov chain $\eta$ visits $u_{1}$ during its first $n$ steps. Thus, while $c(t)$ is the number of visits of $\eta$ to $u_{1}$ up to time $t>0$ on the clock of the random walk, $v(n)$ is the number of occurrences of $u_{1}$ among the first $n$ directions of the random walk. In particular, $v\left(N_{t}\right)=c(t)$. Taking into account (3.2), the law of large numbers and the renewal theorem imply that

$$
\lim _{n \rightarrow \infty} \frac{\tau_{n}}{n}=\lim _{n \rightarrow \infty} \frac{n}{v(n)}=E\left(\tau_{2}-\tau_{1}\right)=\pi_{1}^{-1}, \quad \text { a.s. }
$$

and, letting $\Lambda_{k}:=\sum_{i=\tau_{k}+1}^{\tau_{k+1}} \eta_{i}$

$$
\mu=\lim _{n \rightarrow \infty} \frac{\sum_{i=1}^{n} \eta_{i}}{n}=\lim _{n \rightarrow \infty} \frac{\sum_{k=0}^{v(n)} \Lambda_{k}}{n}=\pi_{1} \cdot E\left(\Lambda_{1}\right), \quad \text { a.s. }
$$

Since $\eta$ and $\left(T_{k}\right)_{k \in \mathbb{N}}$ are independent, it follows that

$$
E\left(\xi_{1}\right)=E\left(T_{1}\right) \cdot E\left(\Lambda_{1}\right)=\pi_{1}^{-1} \mu \cdot E\left(T_{1}\right) .
$$

Finally, $\frac{c(t)}{t}=\frac{v\left(N_{t}\right)}{t}=\frac{v\left(N_{t}\right)}{N_{t}} \cdot \frac{N_{t}}{t}$ yields

$$
\lim _{t \rightarrow \infty} \frac{c(t)}{t}=\frac{\pi_{1}}{E\left(T_{1}\right)}, \quad \text { a.s. }
$$

We now turn to the proofs of our main results.

\subsection{Proof of Theorem 2.1.}

Part (a) of Theorem 2.1. Recall (3.2) and observe that the moment condition of the theorem along with the independence of the Markov chain $\eta$ and $\left(T_{k}\right)_{k \in \mathbb{N}}$ of each other implies that

$$
\begin{aligned}
E\left(\left\|\xi_{1}\right\|^{p}\right) & \leq E\left[\left(s_{\tau_{2}}-s_{\tau_{1}}\right)^{p}\right]=\sum_{n=1}^{\infty} P\left(\tau_{2}-\tau_{1}=n\right) \cdot E\left[\left(\sum_{k=1}^{n} T_{k}\right)^{p}\right] \\
& \leq K_{1} \sum_{n=1}^{\infty} e^{-K_{2}(n-1)} n^{p} E\left(T_{1}^{p}\right)<\infty,
\end{aligned}
$$

where we used Minkowski's inequality and (3.2). It follows that $\left\|\xi_{k}\right\|=\mathrm{o}\left(k^{1 / p}\right)$. Indeed, for any $\varepsilon>0$, Chebyshev's inequality implies that

$$
\sum_{k=1}^{\infty} P\left(\left\|\xi_{k}\right\|>k^{\frac{1}{p}} \varepsilon\right)=\sum_{k=1}^{\infty} P\left(\left\|\xi_{k}\right\|^{p}>\varepsilon^{p} k\right) \leq \varepsilon^{-p} E\left(\left\|\xi_{1}\right\|^{p}\right)<\infty
$$

and hence $P\left(\left\|\xi_{k}\right\|>k^{\frac{1}{p}} \varepsilon\right.$ i.o. $)=0$ by the Borel-Cantelli lemma. 
For now we will make a simplifying assumption (to be removed later on) that $\mu=0$. By virtue of (3.4), the Marcinkiewicz-Zigmund law of large numbers implies that

$$
\lim _{t \rightarrow \infty} \frac{\sum_{i=0}^{c(t)-1} \xi_{i}}{t^{1 / p}}=0, \quad \text { a.s. }
$$

Furthermore, by (3.1), $\left\|X_{t}-\sum_{i=0}^{c(t)-1} \xi_{i}\right\| \leq r_{c(t)}$, where

$$
r_{k}:=\sum_{i=\tau_{k}+1}^{\tau_{k+1}} T_{i}
$$

An argument similar to the one which we used to estimate the order of $\left\|\xi_{n}\right\|$ shows that with probability one, $r_{n}=o\left(n^{1 / p}\right)$. Then (3.4) implies that

$$
r_{c(t)}=o\left(t^{1 / p}\right) \text {. }
$$

This completes the proof of part (a) of Theorem 2.1 for the particular case $\mu=0$.

We now turn to the general case of arbitrary finite $\mu \in \mathbb{R}^{d}$. Let

$$
\tilde{\eta}_{i}=\eta_{i}-\mu \quad \text { and } \quad \tilde{X}_{t}=\sum_{i=0}^{N_{t}} T_{i} \tilde{\eta}_{i}+\left(t-s_{N_{t}}\right) \tilde{\eta}_{N_{t}} .
$$

Then $\widetilde{X}_{t}$ is a directionally reinforced random walk associated with $\left(T_{n}\right)_{n \in \mathbb{N}}$ and $\tilde{\eta}=\left(\tilde{\eta}_{n}\right)_{n \in \mathbb{N}}$. Since $E\left(\tilde{\eta}_{i}\right)=0$, we have $\left\|\widetilde{X}_{t}\right\|=o\left(t^{1 / p}\right)$. To complete the proof of part (a) of the theorem, observe that $X_{t}-\widetilde{X}_{t}=\mu \cdot \sum_{i=1}^{N_{t}} T_{i}+\mu \cdot\left(t-s_{N_{t}}\right)=\mu t$.

Part (b) of Theorem 2.1. Recall (3.3). Let

$$
\bar{\xi}_{k}:=\xi_{k}-E\left(\xi_{1}\right)=\xi_{k}-E\left(T_{1}\right) \pi_{1}^{-1} \mu
$$

and

$$
\Delta_{k}:=s_{\tau_{k+1}-1}-s_{\tau_{k}-1}-E\left(T_{1}\right) \pi_{1}^{-1} .
$$

Let $\gamma_{k}=\left(\bar{\xi}_{k}, \Delta_{k}\right) \in \mathbb{R}^{d+1}$. Then $\left(\gamma_{k}\right)_{k \geq 1}$ is an i.i.d. sequence with $E\left(\gamma_{1}\right)=0 \in$ $\mathbb{R}^{d+1}$. Define

$$
\Gamma(t)=\sum_{1 \leq k \leq t} \gamma_{k}
$$

By virtue of Theorem 1.2.1 in [9], there is a Brownian motion $(B(t))_{t \geq 0}$ in $\mathbb{R}^{d+1}$ such that

$$
\sup _{0 \leq t \leq T}\|\Gamma(t)-B(t)\|=o\left(T^{1 / p}\right) .
$$

Then Theorem 2.3.6 in 9] implies that there exists a Brownian motion $(W(t))_{t \geq 0}$ such that

$$
\sup _{0 \leq t \leq T}\left\|\sum_{k=0}^{c(t)-1} \xi_{k}-t \mu-W(t)\right\|=o\left(T^{1 / p}\right) .
$$

Recall $r_{k}$ from (3.6). Since

$$
\sup _{0 \leq t \leq T}\left\|X_{t}-\sum_{k=0}^{c(t)-1} \xi_{k}\right\| \leq \sup _{0 \leq t \leq T} r_{c(t)},
$$


it suffices to show that

$$
\sup _{0 \leq t \leq T} r_{c(t)}=o\left(T^{1 / p}\right)
$$

Notice that

$$
\sup _{0 \leq t \leq T} r_{c(t)}=\sup _{0 \leq k \leq c(T)} r_{k} .
$$

Therefore, by virtue of (3.4), it suffices to show that

$$
\lim _{n \rightarrow \infty} n^{-1 / p} \cdot \sup _{0 \leq k \leq n} r_{k}=0, \quad \text { a.s. }
$$

Toward this end, let

$$
g(n)=\max \left\{k \leq n: r_{k} \geq r_{i} \text { for all } 1 \leq i \leq n\right\}, \quad n \in \mathbb{N} .
$$

Thus $g(n) \leq n$ and $\sup _{0 \leq k \leq n} r_{k}=r_{g(n)}$. Furthermore, since $r_{k}$ are i.i.d. random variables, $\lim _{n \rightarrow \infty} g(n)=\infty$ with probability one. Therefore, $r_{n}=o\left(n^{1 / p}\right)$ yields (3.10). The proof of Theorem 2.1 is completed.

\subsection{Proof of Theorem 2.3 .}

Part (a) of Theorem 2.3. By (3.5), $\left.E\left(\left\|\xi_{1}\right\|^{2}\right)\right)<\infty$ under the conditions of the theorem. Assume first that $\mu=0$. Then the invariance principle for i.i.d. sequences implies that

$$
\frac{\sum_{k=1}^{[n t]} \xi_{k}}{\sqrt{n}} \Rightarrow W(t), \quad t \in[0,1],
$$

where $W(t)$ is a $d$-dimensional Brownian motion. It follows then from (3.4) and Theorem 14.4 in [6, p. 152] that

$$
\frac{\sum_{k=0}^{c(n t)-1} \xi_{k}}{\sqrt{n}} \Rightarrow \sqrt{b} \cdot W(t), \quad t \in[0,1],
$$

where $b=\frac{\pi_{1}}{\cdot E\left(T_{1}\right)}$. Under the moment condition of Theorem 2.3 we have the following counterpart of (3.9):

$$
\sup _{0 \leq t \leq T} r_{c(t)}=o\left(T^{1 / 2}\right)
$$

Since $\left\|X_{n t}-\sum_{k=0}^{c(n t)-1} \xi_{k}\right\|$ is bounded above by $r_{c(n t)}$, it follows that

$$
n^{-1 / 2} \cdot\left\|X_{n t}-\sum_{k=0}^{c(n t)-1} \xi_{k}\right\| \Rightarrow 0,
$$

which implies the desired convergence of $n^{-1 / 2} \cdot X_{n t}$ when $\mu=0$. To prove the general case of arbitrary $\mu \in \mathbb{R}^{d}$ one can apply the result with $\mu=0$ to the Markov chain $\tilde{\eta}_{n}$ and the random walk $\tilde{X}_{t}$ that were introduced in (3.8). The proof of part (a) of the theorem is completed.

Part (b) of Theorem 2.3. Suppose first that $\mu=0$. For $x \in \operatorname{Span}(U) \subset \mathbb{R}^{d}$ and $i \in \mathbb{N}$ define

$$
\xi_{i, x}:=\xi_{i} \cdot x
$$


Then, in view of (3.4), the law of iterated logarithm for i.i.d. sequences implies that there exists a constant $K(x) \in(0, \infty)$ such that

$$
\limsup _{t \rightarrow \infty} \frac{\sum_{i=0}^{c(t)-1} \xi_{i, x}}{\sqrt{t \ln \ln t}}=K(x), \quad \text { a.s. }
$$

By (3.3) and (3.7)

$$
\lim _{t \rightarrow \infty} \frac{\left|X_{t} \cdot x-\sum_{i=0}^{c(t)-1} \xi_{i, x}\right|}{\sqrt{t \ln \ln t}}=0, \quad \text { a.s. }
$$

Thus

$$
\limsup _{t \rightarrow \infty} \frac{X_{t} \cdot x}{\sqrt{t \ln \ln t}}=K(x), \quad \text { a.s. }
$$

in the case $\mu=0$. To obtain the general case with an arbitrary $\mu \in \mathbb{R}^{d}$, apply this result to the random walk $\widetilde{X}_{t}$ defined in (3.8) and recall that $X_{t}-\widetilde{X}_{t}=\mu t$. The proof of part (b) of Theorem 2.3 is completed.

\subsection{Proof of Theorem 2.5.}

Part (a)-(i) and part (b) of Theorem 2.5 . Let $\overline{\mathbb{R}}_{0}^{d}:=[-\infty, \infty]^{d} \backslash\{0\}$, where 0 stands for the zero vector in $\mathbb{R}^{d}$, and equip $\overline{\mathbb{R}}_{0}^{d}$ with the topology inherited from $\mathbb{R}^{d}$. Recall (see for instance [4,24]) that a random vector $\xi \in \mathbb{R}^{d}$ is said to be regularly varying with index $\alpha>0$ if there exists a function $a: \mathbb{R}_{+} \rightarrow \mathbb{R}$, regularly varying with index $1 / \alpha$, and a Radon measure $\nu_{\xi}$ on $\overline{\mathbb{R}}_{0}^{d}$ such that

$$
n P\left(a_{n}^{-1} \xi \in \cdot\right) \stackrel{v}{\Rightarrow} \nu_{\xi}(\cdot), \quad \text { as } n \rightarrow \infty,
$$

where $a_{n}:=a(n)$ and $\stackrel{v}{\Rightarrow}$ denotes the vague convergence of measures. We will denote by $\mathcal{R}_{d, \alpha, a}$ the set of all random $d$-vectors regularly varying with index $\alpha$, associated with a given function $a \in \mathcal{R}_{1 / \alpha}$ by (3.13). The measure $\nu$ is referred to as the measure of regular variation associated with $\xi$. We will also use the following equivalent definition of the regular variation for random vectors (see, for instance, [4, 24]). Let $S^{d-1}$ denote the unit sphere in $\mathbb{R}^{d}$ with respect to the norm $\|\cdot\|$. Then $\xi \in \mathcal{R}_{d, \alpha, a}$ if and only if there exists a finite Borel measure $\mathfrak{S}_{\xi}$ on $S^{d-1}$ such that for all $t>0$,

$$
n P\left(\|\xi\|>t a_{n} ; \xi /\|\xi\| \in \cdot\right) \stackrel{v}{\Rightarrow} t^{-\alpha} \mathfrak{S}_{\xi}(\cdot), \quad \text { as } n \rightarrow \infty,
$$

where $\stackrel{v}{\Rightarrow}$ denotes the vague convergence of measures on $S^{d-1}$. The following wellknown result is the key to the proof of the next lemma: If $\xi, \eta \in \mathcal{R}_{d, \alpha, a}$ and $\xi, \eta$ are independent of each other, then $\xi+\eta \in \mathcal{R}_{d, \alpha, a}$. Furthermore, $\nu_{\xi+\eta}=\nu_{\xi}+\nu_{\eta}$ and $\mathfrak{S}_{\xi+\eta}=\mathfrak{S}_{\xi}+\mathfrak{S}_{\eta}$ (see, for instance, Equation 1.15 and Lemma 2.6 in [8]).

We have:

Lemma 3.1. Let Assumption 2.4 hold. For $t \geq 0$, let $a_{t}$ be defined as in (2.3). Then

(a) $\sum_{\tau_{1}+1}^{\tau_{2}} T_{i} \in \mathcal{R}_{1, \alpha, a}$.

(b) $\xi_{1} \in \mathcal{R}_{d, \alpha, a}$.

Proof of Lemma 3.1. The claim of part (a) can be formally deduced from that of part (b). Thus we will focus on proving the more general claim (b). 
First, observe that (3.14) implies that $T_{1} u \in \mathcal{R}_{d, \alpha, a}$ for any $u \in U$. Let

$$
H(u, v)=P\left(\eta_{n+1}=v \mid \eta_{n}=u\right), \quad u, v \in U,
$$

be the transition matrix of the Markov chain $\eta$. Further, define a sub-Markovian kernel $\Theta$ by setting

$$
\Theta(u, v)=H(u, v) \cdot \mathbf{1}_{\left\{v \neq u_{1}\right\}}, \quad u, v \in U .
$$

Fix any $t>0$ and a Borel set $B \subset S^{d-1}$, and let

$$
A_{n}=\left\{\left\|\xi_{1}\right\|>t a_{n} ; \xi_{1} /\left\|\xi_{1}\right\| \in B\right\}, \quad n \in \mathbb{N} .
$$

Then,

$$
\begin{aligned}
& P\left(\xi_{1} \in A_{n}\right)= \sum_{k=1}^{\infty} P\left(\tau_{2}-\tau_{1}=k\right) \\
& \times P\left(T_{1} u_{1}+T_{2} \eta_{2}+\ldots+T_{k} \eta_{k} \in A_{n} \mid \tau_{2}-\tau_{1}=k\right) \\
&=\sum_{k=1}^{\infty} \sum_{v_{2} \neq u_{1}} \cdots \sum_{v_{k} \neq u_{1}} \Theta\left(u_{1}, v_{1}\right) \cdots \Theta\left(v_{k-1}, v_{k}\right) H\left(v_{k}, u_{1}\right) \\
& \times P\left(T_{1} u_{1}+T_{2} v_{2}+\ldots+T_{k} v_{k} \in A_{n}\right),
\end{aligned}
$$

where we assume that the sums $\sum_{v_{2} \neq u_{1}} \cdots \sum_{v_{k} \neq u_{1}}$ are empty if $k=1$. Let

$$
J_{n}\left(v_{2}, \ldots, v_{k}\right)=T_{1} u_{1}+T_{2} v_{2}+\ldots+T_{k} v_{k}
$$

Notice that for any $k \in \mathbb{N}$ and fixed set of vectors $v_{2}, \ldots, v_{k} \in U$, we have

$$
\begin{aligned}
& n \cdot P\left(J_{n}\left(v_{2}, \ldots, v_{k}\right) \in A_{n}\right) \leq n \cdot P\left(\left\|J_{n}\left(v_{2}, \ldots, v_{k}\right)\right\| \geq t a_{n}\right) \\
& \quad \leq n P\left(\sum_{j=1}^{k} T_{j} \geq t a_{n}\right) \leq n k P\left(T_{1} \geq t a_{n} / k\right) \leq C t^{-\alpha} k^{1+\alpha}
\end{aligned}
$$

for some $C>0$. Furthermore,

$$
\lim _{n \rightarrow \infty} n \cdot P\left(J_{n}\left(v_{2}, \ldots, v_{k}\right) \in A_{n}\right)=t^{-\alpha}\left(\mathfrak{S}_{T_{1} u_{1}}(B)+\sum_{j=2}^{k} \mathfrak{S}_{T_{1} v_{j}}(B)\right) .
$$

Observe that the spectral radius of the matrix $\Theta$ is strictly less than one and that $\mathfrak{S}_{T_{1} v_{j}}(B)$ is uniformly bounded from above by $\max _{v \in U} \mathfrak{S}_{T_{1} v}\left(S^{d-1}\right)$. Therefore, the dominated convergence theorem implies that the following limit exists and the identity holds:

$$
\begin{aligned}
& \lim _{n \rightarrow \infty} n \cdot P\left(\xi_{1} \in A_{n}\right) \\
&=\sum_{k=1}^{\infty} t^{-\alpha} \sum_{v_{2} \neq u_{1}} \cdots \sum_{v_{k} \neq u_{1}} \Theta\left(u_{1}, v_{1}\right) \cdots \Theta\left(v_{k-1}, v_{k}\right) H\left(v_{k}, u_{1}\right) \\
& \times\left(\mathfrak{S}_{T_{1} u_{1}}(B)+\sum_{j=2}^{k} \mathfrak{S}_{T_{1} v_{j}}(B)\right) .
\end{aligned}
$$

Since the spectral radius of $\Theta$ is strictly less than one, Fubini's theorem implies that the right-hand side of the above identity defines a measure on $S^{d-1}$. The proof of the lemma is therefore completed. 
We are now in a position to complete the proof of the limit results stated in parts (a) and (b) of Theorem 2.5. Suppose first that $\mu=0$. It follows from Lemma 3.1 and the stable limit theorem for i.i.d. sequences (see, for instance, Section 1.6 in [7, p. 75]) that

$$
\frac{\sum_{k=1}^{[n t]} \xi_{k}}{a_{n}} \Rightarrow S_{\alpha}(t), \quad t \in[0,1],
$$

where $S_{\alpha}(t)$ is a homogeneous vector-valued process in $D\left(\mathbb{R}^{d}\right)$ with independent increments and $S_{\alpha}(1)$ distributed according to a stable law of index $\alpha$. Then (similarly to (3.11) ), asymptotic equivalence (3.4) along with the suitable modification of Theorem 14.4 in [6, p. 152] implies

$$
\frac{\sum_{k=0}^{c([n t])-1} \xi_{k}}{a_{n}} \Rightarrow b^{1 / \alpha} \cdot S_{\alpha}(t), \quad t \in[0,1],
$$

where $b=\frac{\pi_{1}}{\cdot E\left(T_{1}\right)}$. In particular, using $t=1$,

$$
\frac{\sum_{k=0}^{c(n)-1} \xi_{k}}{a_{n}} \Rightarrow b^{1 / \alpha} \cdot S_{\alpha}(1) .
$$

Recall $r_{k}$ from (3.6). Since

$$
\left\|X_{t}-\sum_{k=0}^{c(t)-1} \xi_{k}\right\| \leq r_{c(t)},
$$

an application of the renewal theorem shows that

$$
\frac{X_{n}}{n} \Rightarrow \mathcal{L}_{\alpha}, \quad \text { and hence } \quad \frac{X_{\lfloor t\rfloor}}{t} \Rightarrow \mathcal{L}_{\alpha}
$$

Since $\left\|X_{\lfloor t\rfloor}-X_{t}\right\| \leq 1$, the proof of part (a)-(i) of Theorem 2.5 is completed.

Part (a)-(ii) of Theorem 2.5. For $v \in U$ let $c_{v}(t)$ be the number of occurrences of $v$ in the set $\left\{\eta_{1}, \eta_{2}, \ldots, \eta_{N_{t}}\right\}$. That is,

$$
c_{v}(t)=\sum_{k=1}^{N_{t}} \mathbf{1}_{\left\{\eta_{k}=v\right\}}, \quad t \geq 0, v \in U .
$$

Notice that $c_{u_{1}}(t)=c(t)$, where $c(t)$ was introduced in Section 3.1 Similarly to (3.4) we have

$$
\lim _{t \rightarrow \infty} \frac{c_{v}(t)}{t}=\frac{\pi_{v}}{E\left(T_{1}\right)}, \quad \text { a.s. }
$$

where $\pi_{v}$ is the mass that the stationary distribution of the Markov chain $\eta$ puts on $v$.

Define $\tau_{v}(0)=0$ and $\tau_{v}(j)=\inf \left\{k>\tau_{v}(j-1): \eta_{k}=v\right\}$ for $j \in \mathbb{N}$. For $v \in U$ and $t \geq 0$, let

$$
\widetilde{B}_{v}(t)=\sum_{i=0}^{c_{v}(t)-1} T_{\tau_{v}(i)}-c_{v}(t) \cdot E\left(T_{1}\right) .
$$


Then, the law of iterated logarithm for heavy-tailed i.i.d. sequences (see Theorems 1.6.6 and 3.9.1 in [7]) combined with (3.4) yields

$$
\limsup _{t \rightarrow \infty} \frac{\widetilde{B}_{v}(t)}{a_{t} \cdot(\ln t)^{1 / \alpha+\varepsilon}}=\left\{\begin{array}{ll}
0 & \text { if } \varepsilon>0, \\
\infty & \text { if } \varepsilon<0
\end{array} \quad\right. \text { a.s. }
$$

For $v \in U$, let

$$
B_{v}(t)=\sum_{i=0}^{c_{v}(t)-1}\left(T_{\tau_{v}(i)}-E\left(T_{1}\right)\right)+\left(t-s_{N_{t}}\right) \mathbf{1}_{\left\{\eta_{N_{t}}=v\right\}} .
$$

Then, (1.1) implies that

$$
X_{t}=\sum_{v \in U} v B_{v}(t)+\left(\sum_{v \in U} v \cdot c_{v}(t) \cdot E\left(T_{1}\right)-\mu \cdot t\right) .
$$

Taking into account (3.17), a standard inversion argument allows one to deduce from the law of iterated logarithm for $\tau_{v}(n)$ that

$$
\limsup _{t \rightarrow \infty} \frac{\left\|\sum_{v \in U} v \cdot c_{v}(t) \cdot E\left(T_{1}\right)-\mu \cdot t\right\|}{\sqrt{t \ln \ln t}}<\infty, \quad \text { a.s. }
$$

Since $a_{t} \in \mathcal{R}_{\alpha}$ with $\alpha \in(1,2)$,

$$
\lim _{t \rightarrow \infty} \frac{\sqrt{t \ln \ln t}}{a_{t} \cdot(\ln t)^{1 / \alpha+\varepsilon}}=0 .
$$

Thus (3.18) along with (3.19) yields part (a)-(ii) of Theorem 2.5, provided that we are able to show that for any $u, v \in U$ and all $\delta \in(1 /(2 \alpha), 1 / \alpha)$,

$$
P\left(E_{n, v, \delta} \cap E_{n, u, \delta} \text { i.o. }\right)=0,
$$

where the events $E_{n, v, \delta}$ are defined for $n \in \mathbb{N}, v \in U$, and $\delta \in(1 /(2 \alpha), 1 / \alpha)$ as follows:

$$
E_{n, v, \delta}:=\left\{\max _{1 \leq m \leq c_{v}(n)}\left|\sum_{i=0}^{m-1} T_{\tau_{v}(i)}-m \cdot E\left(T_{1}\right)\right|>a_{n} \cdot(\ln n)^{\delta}\right\} .
$$

For $n \in \mathbb{N}$ let $\gamma_{n}=2 n \cdot \max _{v \in V} \pi_{v}$ and define

$$
G_{n, v, \delta}:=\left\{\max _{1 \leq m \leq \gamma_{n}}\left|\sum_{i=0}^{m-1} T_{\tau_{v}(i)}-m \cdot E\left(T_{1}\right)\right|>a_{n} \cdot(\ln n)^{\delta}\right\} .
$$

Now fix any directions $u, v \in U$ and a constant $\delta \in(1 /(2 \alpha), 1 / \alpha)$. We have

$$
\begin{aligned}
& P\left(E_{n, v, \delta} \cap E_{n, u, \delta}\right) \\
& \quad \leq P\left(G_{n, v, \delta} \cap G_{n, u, \delta}\right)+P\left(c_{v}(n)>\gamma_{n}\right)+P\left(c_{u}(n)>\gamma_{n}\right) \\
& \quad=P\left(G_{n, v, \delta}\right) \cdot P\left(G_{n, u, \delta}\right)+P\left(c_{v}(n)>\gamma_{n}\right)+P\left(c_{u}(n)>\gamma_{n}\right) .
\end{aligned}
$$

It follows from the large deviation principle for the random sequence $c_{v}(n) / n$ that $P\left(c_{v}(n)>\gamma_{n}\right)<K_{v} e^{-n \lambda_{v}}$ for some $K_{v}>0$ and $\lambda_{v}>0$. Furthermore, for any $A>0$ and $k_{n}=\left[A^{n}\right]$, we have $P\left(G_{k_{n}, v, \delta}\right) \leq C n^{-\beta}$ for some constants $\beta=\beta(\delta)>1 / 2$ and $C>0$ (see [7, p. 177]; here we exploit the constraint $2 \alpha \delta>1$ ). The Borel-Cantelli lemma then implies that $P\left(G_{k_{n}, v, \delta} \cap G_{k_{n}, u, \delta}\right.$ i.o. $)=0$. Since for any $n \in \mathbb{N}$ there is a unique $j(n) \in \mathbb{N}$ such that $k_{j(n)} \leq n<k_{j(n)+1}$, and $\lim _{k \rightarrow \infty} \frac{a_{k+1}\left(\ln a_{k+1}\right)^{\delta}}{a_{k}\left(\ln a_{k}\right)^{\delta}}=1$, this yields (3.20). The proof of part (a)-(ii) of Theorem 2.5 is therefore completed. 
3.5. Proof of Theorem 2.6. Define two sequences of processes in $D(\mathbb{R}),\left(B_{n}\right)_{n \in \mathbb{N}}$ and $\left(C_{n}\right)_{n \in \mathbb{N}}$ by setting

$$
B_{n}(t):=\frac{\sum_{k=1}^{[n t]} \xi_{k}}{a_{n}} \quad \text { and } \quad C_{n}(t):=\frac{s_{\tau_{[n t]}}}{a_{n}}, \quad t \in[0,1] .
$$

Lemma 3.1] combined with [4, Theorem 1.1] implies that $\left(\xi_{1}, s_{\tau_{2}}-s_{\tau_{1}}\right) \in \mathcal{R}_{d+1, \alpha, a}$, and hence

$$
\left(B_{n}, C_{n}\right) \Rightarrow\left(S_{\alpha}, U_{\alpha}\right),
$$

where $S_{\alpha}$ and $U_{\alpha}$ are homogeneous processes with independent increments in $D\left(\mathbb{R}^{d}\right)$ and $D(\mathbb{R})$, respectively, such that $S_{\alpha}(1)$ and $U_{\alpha}(1)$ have (multivariate in the former case) stable distributions of index $\alpha$.

Let $U_{n}^{-1}$ and $C_{n}^{-1}$ denote the inverse processes of $U_{n}$ and $C_{n}$, respectively. One can define $C_{n}^{-1}$ explicitly as follows:

$$
C_{n}^{-1}(t)=n^{-1} c\left(a_{n} t\right), \quad t \in[0,1] .
$$

Then the same argument as in [13, pp. 380-381] shows that (alternatively, one can use the result of [31])

$$
\left(B_{n}, C_{n}^{-1}\right) \Rightarrow\left(S_{\alpha}, U_{\alpha}^{-1}\right)
$$

in $D\left(\mathbb{R}^{d+1}\right)$. This along with (3.23) implies (see, for instance, [6, p. 151]) that

$$
\frac{\sum_{i=1}^{c\left(a_{n}\right)-1} \xi_{i}}{a_{n}} \Rightarrow \mathcal{L}_{\alpha}
$$

where

$$
\mathcal{L}_{\alpha}:=S_{\alpha}\left(U_{\alpha}^{-1}\right)(1)
$$

Passing to the subsequence $m_{n}=\left\lfloor a_{n}^{-1}\right\rfloor$ and using basic properties of regularly varying functions, we obtain

$$
\frac{\sum_{i=0}^{c(n)-1} \xi_{i}}{n} \Rightarrow \mathcal{L}_{\alpha}
$$

To conclude the proof of the theorem one can use verbatim the argument along the lines following (3.16) in the concluding paragraph of the above proof of part (a)-(i) of Theorem 2.5. Namely, taking into account the inequality

$$
\left\|X_{t}-\sum_{k=0}^{c(t)-1} \xi_{k}\right\| \leq r_{c(t)}
$$

and using the renewal theorem which ensures the weak convergence of $r_{c(t)}$ to a proper random variable, (3.25) yields that $\frac{X_{t}}{t} \Rightarrow \mathcal{L}_{\alpha}$. The proof of Theorem 2.6 is completed.

\section{ACKNOWLEDGEMENTS}

The authors would like to thank the anonymous referee and the associate editor for their very helpful comments and suggestions. 


\section{REFERENCES}

[1] Pieter Allaart and Michael Monticino, Optimal stopping rules for directionally reinforced processes, Adv. in Appl. Probab. 33 (2001), no. 2, 483-504, DOI 10.1239/aap/999188325. MR.1843184 (2002e:60067)

[2] Pieter Allaart and Michael Monticino, Optimal buy/sell rules for correlated random walks, J. Appl. Probab. 45 (2008), no. 1, 33-44, DOI 10.1239/jap/1208358949. MR2409308 (2009g:60057)

[3] K. B. Athreya and P. Ney, A new approach to the limit theory of recurrent Markov chains, Trans. Amer. Math. Soc. 245 (1978), 493-501, DOI 10.2307/1998882. MR511425(80i:60092)

[4] Bojan Basrak, Richard A. Davis, and Thomas Mikosch, A characterization of multivariate regular variation, Ann. Appl. Probab. 12 (2002), no. 3, 908-920, DOI 10.1214/aoap/1031863174. MR:1925445 (2003h:60022)

[5] Peter Becker-Kern, Mark M. Meerschaert, and Hans-Peter Scheffler, Limit theorems for coupled continuous time random walks, Ann. Probab. 32 (2004), no. 1B, 730-756, DOI 10.1214/aop/1079021462. MR2039941 (2004m:60092)

[6] Patrick Billingsley, Convergence of probability measures, 2nd ed., Wiley Series in Probability and Statistics: Probability and Statistics. A Wiley-Interscience Publication, John Wiley \& Sons Inc., New York, 1999. MR1700749(2000e:60008)

[7] A. A. Borovkov and K. A. Borovkov, Asymptotic analysis of random walks, Heavy-tailed distributions. Translated from the Russian by O. B. Borovkova, Encyclopedia of Mathematics and its Applications, vol. 118, Cambridge University Press, Cambridge, 2008. MR2424161 (2010b:60132)

[8] Dariusz Buraczewski, Ewa Damek, and Mariusz Mirek, Asymptotics of stationary solutions of multivariate stochastic recursions with heavy tailed inputs and related limit theorems, Stochastic Process. Appl. 122 (2012), no. 1, 42-67, DOI 10.1016/j.spa.2011.10.010. MR.2860441 (2012i:60145)

[9] Miklós Csörgő and Lajos Horváth, Weighted approximations in probability and statistics, with a foreword by David Kendall. Wiley Series in Probability and Mathematical Statistics: Probability and Mathematical Statistics, John Wiley \& Sons Ltd., Chichester, 1993. MR 1215046 (94c:60060)

[10] S. Goldstein, On diffusion by discontinuous movements, and on the telegraph equation, Quart. J. Mech. Appl. Math. 4 (1951), 129-156. MR0047963 (13,960b)

[11] A. De Gregorio, On random fights with non-uniformly distributed directions, preprint is available at http://arxiv.org/abs/1110.3621.

[12] Urs Gruber and Martin Schweizer, A diffusion limit for generalized correlated random walks, J. Appl. Probab. 43 (2006), no. 1, 60-73, DOI 10.1239/jap/1143936243. MR2225050 (2007b:60085)

[13] Lajos Horváth and Qi-Man Shao, Limit distributions of directionally reinforced random walks, Adv. Math. 134 (1998), no. 2, 367-383, DOI 10.1006/aima.1997.1707. MR1617789 (99g:60070)

[14] A. Jurlewicz, P. Kern, M. M. Meerschaert, and H.-P. Scheffler, Fractional governing equations for coupled random walks, Comput. Math. Appl. 64 (2012), no. 10, 3021-3036, DOI 10.1016/j.camwa.2011.10.010. MR2989331

[15] Mark Kac, A stochastic model related to the telegrapher's equation, Rocky Mountain J. Math. 4 (1974), 497-509. Reprinting of an article published in 1956. Papers arising from a Conference on Stochastic Differential Equations (Univ. Alberta, Edmonton, Alta., 1972). MR0510166 (58 \#23185)

[16] James E. Kiefer and George H. Weiss, The Pearson random walk, Random walks and their applications in the physical and biological sciences (Washington, D.C., 1982), AIP Conf. Proc., vol. 109, Amer. Inst. Phys., New York, 1984, pp. 11-32, DOI 10.1063/1.34331. MR780190 (86g:60076)

[17] Alexander D. Kolesnik, Random motions at finite speed in higher dimensions, J. Stat. Phys. 131 (2008), no. 6, 1039-1065, DOI 10.1007/s10955-008-9532-0. MR2407379(2009d:60343)

[18] Alexander D. Kolesnik and Enzo Orsingher, A planar random motion with an infinite number of directions controlled by the damped wave equation, J. Appl. Probab. 42 (2005), no. 4, 11681182, DOI 10.1239/jap/1134587824. MR2203830(2007a:60046) 
[19] Aimé Lachal, Cyclic random motions in $\mathbb{R}^{d}$-space with $n$ directions, ESAIM Probab. Stat. 10 (2006), 277-316 (electronic), DOI 10.1051/ps:2006012. MR2247923 (2007k:60036)

[20] R. Daniel Mauldin, Michael Monticino, and Heinrich von Weizsäcker, Directionally reinforced random walks, Adv. Math. 117 (1996), no. 2, 239-252, DOI 10.1006/aima.1996.0011. MR 1371652 (97b:60067)

[21] Mark M. Meerschaert and Hans-Peter Scheffler, Triangular array limits for continuous time random walks, Stochastic Process. Appl. 118 (2008), no. 9, 1606-1633, DOI 10.1016/j.spa.2007.10.005. MR2442372(2010b:60135)

[22] E. Orsingher and A. De Gregorio, Random flights in higher spaces, J. Theoret. Probab. 20 (2007), no. 4, 769-806, DOI 10.1007/s10959-007-0093-y. MR2359055(2008h:60041)

[23] K. Pearson, The problem of the random walk, Nature 72 (1905), 294.

[24] Sidney Resnick, On the foundations of multivariate heavy-tail analysis, J. Appl. Probab. 41A (2004), 191-212, DOI 10.1239/jap/1082552199. MR2057574

[25] Rainer Siegmund-Schultze and Heinrich von Weizsäcker, Level crossing probabilities. II. Polygonal recurrence of multidimensional random walks, Adv. Math. 208 (2007), no. 2, 680698, DOI 10.1016/j.aim.2006.03.009. MR2304333 (2008i:60080)

[26] W. Stadje, The exact probability distribution of a two-dimensional random walk, J. Statist. Phys. 46 (1987), no. 1-2, 207-216, DOI 10.1007/BF01010341. MR887245 (89b:60165)

[27] P. Straka and B. I. Henry, Lagging and leading coupled continuous time random walks, renewal times and their joint limits, Stochastic Process. Appl. 121 (2011), no. 2, 324-336, DOI 10.1016/j.spa.2010.10.003. MR2746178 (2012a:60136)

[28] D. Szász and B. Tóth, Persistent random walks in a one-dimensional random environment, J. Statist. Phys. 37 (1984), no. 1-2, 27-38, DOI 10.1007/BF01012903. MR774883(86e:60056)

[29] Bálint Tóth, Persistent random walks in random environment, Probab. Theory Relat. Fields 71 (1986), no. 4, 615-625, DOI 10.1007/BF00699043. MR833271 (87f:60107)

[30] B. J. West, Statistics of deep water surface waves. In Encyclopedia of Fluid Mechanics, Vol. 2: Dynamics of Single Fluid Flows and Mixing (N. P. Cheremesinoff, ed.), Gulf, Houston, 1986, pp. 26-46.

[31] Ward Whitt, Weak convergence of first passage time processes, J. Appl. Probability 8 (1971), 417-422. MR0307335(46 \#6455)

Department of Statistics, Iowa State University, Ames, Iowa 50011

E-mail address: apghosh@iastate.edu

Department of Mathematics, Iowa State University, Ames, Iowa 50011

E-mail address: reza.rastegar80@gmail.com

Department of Mathematics, Iowa State University, Ames, Iowa 50011

E-mail address: roiterst@iastate.edu 